Appl. Phys. B 49, 33-38 (1989)

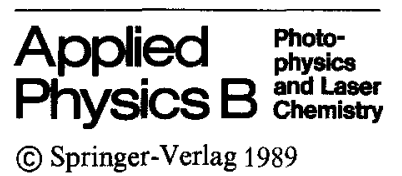

\title{
High-Speed $\mathrm{N}_{2}$-CARS Thermometry
}

\author{
B. Lange ${ }^{1, \star}$, M. Noda ${ }^{1}$, and G. Marowsky ${ }^{2}$ \\ ${ }^{1}$ Nagasaki Research and Development Center, Mitsubishi Heavy Industries, \\ Ltd. 1-2 Akunoura-Machi, 850-91 Nagasaki, Japan \\ ${ }^{2}$ Max-Planck-Institut für biophysikalische Chemie, Abteilung Laserphysik, Am Fassberg, \\ D-3400 Göttingen, Fed. Rep. Germany
}

Received 10 October 1988/Accepted 24 February 1989

\begin{abstract}
Using an excimer laser-pumped CARS spectrometer, single-pulse temperature measurements have been performed with a repetition rate of up to $80 \mathrm{~Hz}$ in a 3-dimensional, phase-matched geometry. Single-pulse temperature data measured in a furnace typically fluctuated by $\pm 50 \mathrm{~K}$ within the temperature range $300-1300 \mathrm{~K}$. The CARS spectra were analyzed by the $G$-matrix formalism with inclusion of cross coherence effects.
\end{abstract}

PACS: 42.65

Coherent anti-Stokes Raman Spectroscopy (CARS) is now widely accepted as a versatile tool for the measurement of temperature $[1,2]$, concentration [3] and even pressure [4]. CARS signals are generated when two phase matched laser beams of frequency $\omega_{p}$ and $\omega_{\mathrm{s}}$, respectively (usually called pump- and Stokesbeam) interact through the nonlinear third order susceptibility $\chi^{(3)}$ of the medium, producing coherent radiation at frequency $\omega_{\mathrm{as}}=2 \omega_{\mathrm{p}}-\omega_{\mathrm{s}}$. Upon resonant excitation of vibronically excited levels, their relative population densities can be measured. Assuming a Boltzmann distribution between these levels, a temperature can be attributed to the ro-vibronic manifold under consideration.

Usually CARS-temperature measurements are based on an evaluation of the $Q$-branch of molecular nitrogen $\left(\mathrm{N}_{2}\right)$. This species is (especially in air-fed combustion) almost everywhere present and, due to the relatively large Raman cross section, the CARSsignal intensity is sufficient for single-pulse measurements. Additionally the simple diatomic structure and a detailed knowledge of spectroscopic constants and

\footnotetext{
* On leave from Max-Planck-Institut für biophysikalische Chemie, Abteilung Laserphysik, Am Fassberg, D-3400 Göttingen, Fed. Rep. Germany; permanent address: Physikalisch-Chemisches Institut, Universität, Im Neuenheimer Feld 253, D-6900 Heidelberg, Fed. Rep. Germany
}

linewidth data result in reliable theoretical calculations of the CARS spectra of nitrogen.

The CARS technique offers high spatial resolution and virtually instantaneous measurements even in hostile environments with high signal intensities and thus preventing problems caused by background radiation. Especially in turbulent media, the high temporal resolution allows monitoring of the fluctuations in both tempeature and concentration. The probability density functions derived from such measurements provide a detailed knowledge of combustion and fluid dynamics. Regarding this, high data aquisition rates are favourable. However the pulse repetition rates for most of the standard CARS systems is determined by the commonly employed Nd:YAG laser (typically 10 or $20 \mathrm{~Hz}$ ) [5-8]. In contrast, with an excimer-laserpumped CARS apparatus, much higher repetition rates can be achieved so that even for short time events a statistically sufficient number of data can be measured.

\section{Theory}

The CARS theory is outlined in detail in $[9,10]$. Therefore only a brief description will be given here. Generally, a CARS spectrum (neglecting convolutions) can be computed from the following expression for $\chi^{(3)}$ :

$\chi^{(3)}=\frac{N}{\hbar} \sum_{j} \alpha_{j j} \sum_{k} \alpha_{k k} \Delta \underline{\varrho}_{k}[G]_{j k}^{-1}+\chi^{\mathrm{NR}}$, 
where $N$ is the number of molecules per $\mathrm{cm}^{3}, h=h / 2 \pi, \alpha$ the diagonal matrix of transition amplitudes, $\Delta \varrho$ the diagonal matrix of fractional population differences, $G$ the $G$-matrix and $\chi^{\mathrm{NR}}$ the total nonresonant thirdorder susceptibility of the medium for a given set of pump, Stokes and CARS frequencies. An element of the matrix $\alpha$ can be calculated by

$\alpha_{j k}=\left(\frac{c}{\omega_{\mathrm{s}}}\right)^{2}\left(\frac{d \sigma}{d \Omega}\right)^{1 / 2} \delta(j-k)$

where $\left(\frac{d \sigma}{d \Omega}\right)$ is the differential Raman cross section and $\delta$ the Kronecker delta function. Details of the $G$-matrix formalism can be found in $[9,10]$.

Expression (1) requires the inversion of the matrix $G$ for every spectral point of interest.

Following the method of Koszykowski et al. [11], this can be circumvented by a procedure requiring only one inversion per spectrum but, additionally, the calculation of the (complex) eigenvalues and eigenvectors of the reduced $G$-matrix. The susceptibility $\chi^{(3)}$ finally follows from the expression:

$\chi^{(3)}=\frac{N}{\hbar} \sum_{j} a_{j}\left[\lambda_{j}-\Delta\right]^{-1}+\chi^{\mathrm{NR}}$

with $a_{j}$ as a complex weighting factor (calculated from the eigenvector matrix, the matrix of fractional population differences and the matrix of transition amplitudes), $\lambda_{j}$ the $j$ th eigenvalue of the reduced $G$-matrix and $\Delta=-\omega_{\mathrm{p}}-\omega_{\mathrm{s}}$.

To consider cross-coherence effects, the pump laser convolution must simulate the process of producing frequency degenerate CARS signals from two distinct photons within the spectral profile of the pump laser.
For Gaussian [12] and Lorentzian [13] pump laser lineshapes, analytical expressions for $\chi^{(3)}$ including cross-coherence have been derived. Cross-coherence is particularly important when large nonresonant signal contributions are detected.

The theoretical spectra were calculated by expression (3) and the analytical solution for $\chi^{(3)}$ for Gaussian pump laser profiles. Spectral fits and calculations were performed on a microcomputer (4 Mbyte, 32 bit, $25 \mathrm{MHz})$.

\section{Experimental Setup}

A schematic of the experimental apparatus is given in Fig. 1. The narrow-band pump laser as well as the broadband Stokes laser (Lambda Physik FL 2002 and a prototype FL 1002, respectively) were both pumped with $55 \%$ and $45 \%$, respectively, of the output of an excimer laser (Lambda Physik EMG 201). The laser energies were $350 \mathrm{~mJ}$ at $308 \mathrm{~nm}(\mathrm{XeCl})$ for the excimer laser, $20 \mathrm{~mJ}$ for the pump laser at $480 \mathrm{~nm}$ (operated with the dye Coumarin 153 dissolved in methanol) with a bandwidth of $0.2 \mathrm{~cm}^{-1}$ and $15 \mathrm{~mJ}$ for the Stokes laser at $540 \mathrm{~nm}$ (operated with the dye Coumarin 103 dissolved in methanol) with an adjustable bandwidth of $3-150 \mathrm{~cm}^{-1}$. Both dye lasers showed pulse durations of $15 \mathrm{~ns}$. The dye-solutions were circulated by pumps which provided high flow speed $(110 \mathrm{~cm} / \mathrm{s})$ and a large reservoir of dye-solution (5 liters). Details of the broadband dye laser are given in Fig. 2a. The dispersive element, which determines the centre wavelength and together with the appropriate alignment the spectral range of emitted radiation was a $R=100 \%$ rear-side coated prism (Littrow-prism). The centre

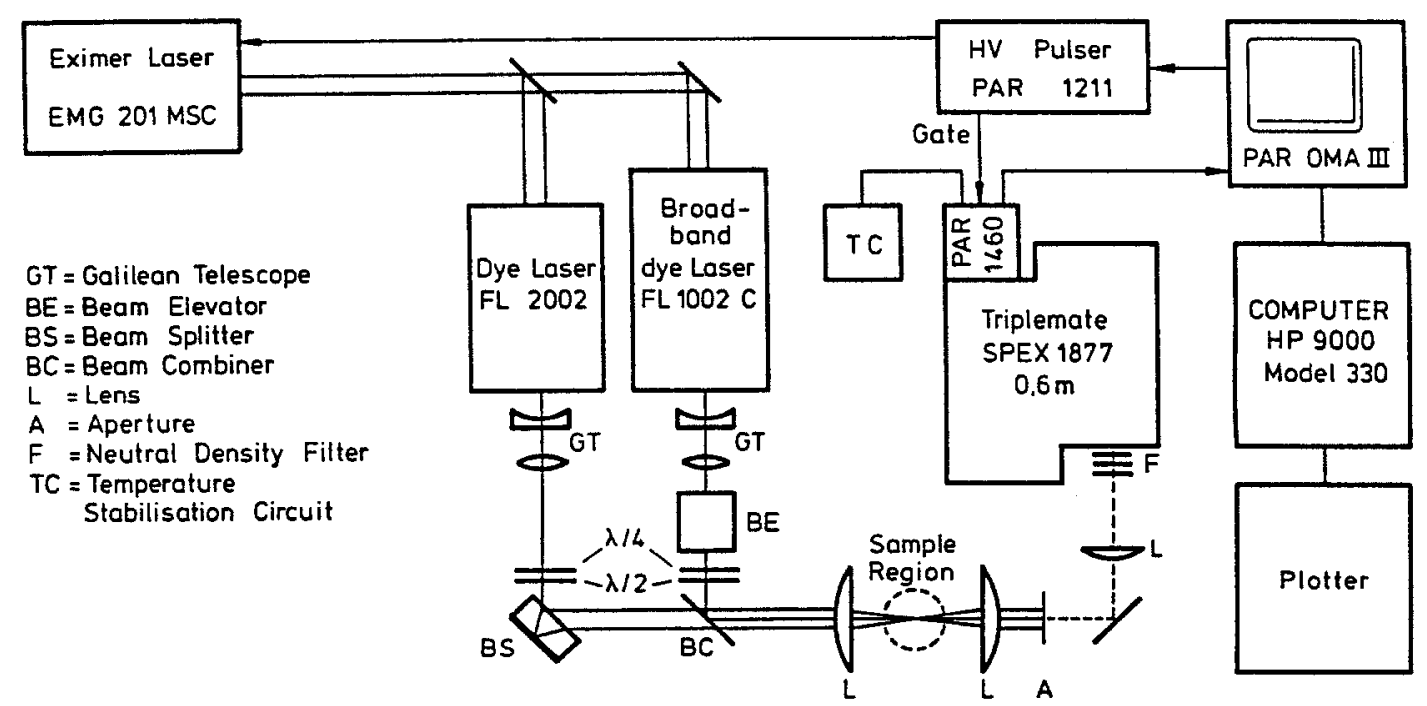

Fig. 1. Experimental setup of excimer laser-pumped dual dye-laser apparatus 


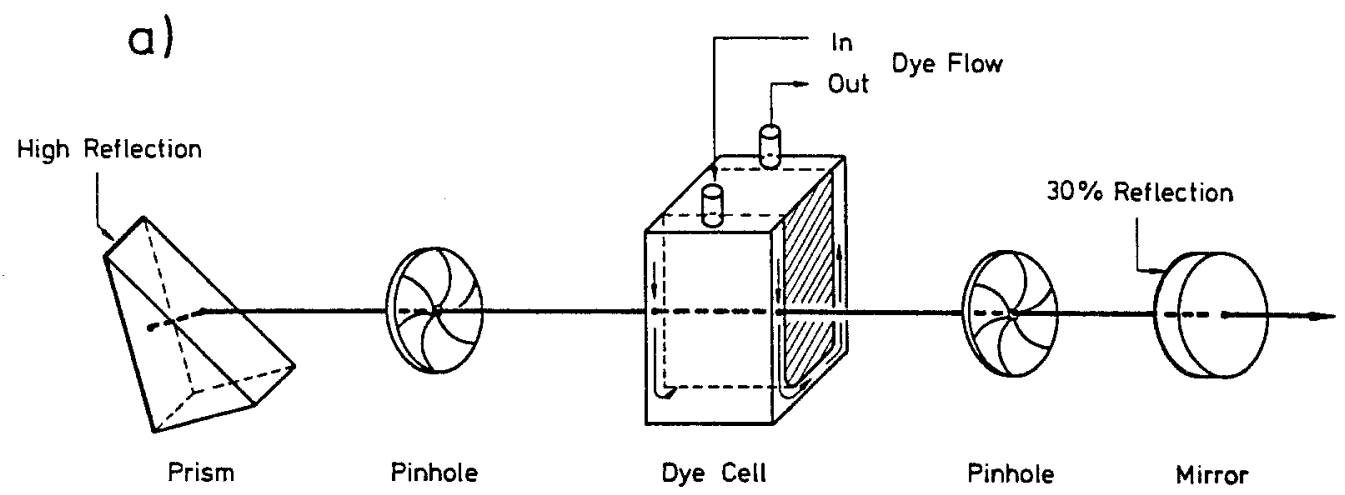

b)

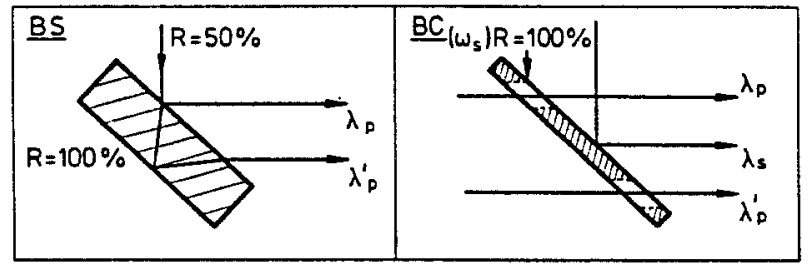

Fig. 2. a Broadband Stokes laser of adjustable bandwidth and tunable centre frequency. b Beam splitter and beam combining mirror for BOXCARS geometry of pump and Stokes beams

wavelength of the broadband Stokes laser can easily be changed by rotating the prism with respect to the optical axis without any change of the spatial beam position and profile. At the Stokes laser wavelength of $540 \mathrm{~nm}$ the angle between oscillator beam axis and hypotenuse of the Littrow-prism was $0.06 \mathrm{rad}$. The Stokes laser oscillator cavity has an optical length of $45 \mathrm{~cm}$. For the measurements reported herein, a BK 7prism was used which resulted in a Stokes bandwidth of $80 \mathrm{~cm}^{-1}$ (FWHM). Polarization devices guaranteed linear and parallel polarizations of pump and Stokes laser light. With Galilean telescopes in both lasers a common focus of all beams was adjusted and the laser beams enlarged about three times to diameters of $1.5 \mathrm{~mm}$. The pump beam was divided by a beamsplitter as shown in Fig. 2b. This beamsplitter introduced an optical delay of $2.6 \mathrm{~cm}$ between the two components of the pump beam. The pump laser had a coherence length of less than $4 \mathrm{~cm}$. By the use of an optical flat we introduced an additional delay of $1.5 \mathrm{~cm}$ so that - to a good approximation - uncorrelated pump photons interacted, which has been shown to be important for a correct evaluation of the measured CARS spectra [14].

Pump and Stokes beams were combined in a BOXCARS-geometry by a beam-collecting mirror (see Fig. 2b) provided with holes for lossless transmission of the pump beams. The CARS signals were generated with a $f=150 \mathrm{~mm}$ lens $(f=300 \mathrm{~mm}$ for furnace measurements) within a cylinder of $50 \mu \mathrm{m}$ diameter and $3 \mathrm{~mm}$ length [15] and focussed onto the slit of a Spex monochromator (Triple-mate). The latter was equipped with a $18001 / \mathrm{mm}$ grating and a gateable optical multichannel analyzer (PAR, OMA III) which was connected to a HP microcomputer by a parallel interface. No further optical elements like colour glass filters or dispersive prisms were needed to reject stray light.

The furnace was a home-made microprocessorcontrolled unit, capable of stable operation for temperatures as high as $1300 \mathrm{~K}$. The heated cell consisted of an alumina tube, furnished with several alumina rings to prevent convection. The cell was closed by O-ring sealed quartz windows. The temperature difference along the CARS probing length was measured by a thermocouple to be less than $10 \mathrm{~K}$ at $1300 \mathrm{~K}$. A turbulent flame was produced by a Bunsen-type burner operated with a premixed stoichiometric methane/air mixture. The burner had a free diameter of $4 \mathrm{~cm}$ in which a grid was mounted to achieve highly turbulent flow conditions of the combustion gases.

\section{Results and Discussion}

\section{Concentration Measurements}

To confirm the validity of our theoretical model, we measured spectra of known mixtures of nitrogen and argon at room temperature and pressure by focussing with $f=150 \mathrm{~mm}$ lenses. With a careful adjustment of the common focus and divergences of pump and Stokes beams, pump-induced population changes were observed [15]. To avoid such population changes, the laser energies were reduced for all subsequent measurements to $10 \mathrm{~mJ}$ for the pump and $13 \mathrm{~mJ}$ for the Stokes laser.

Figure 3 shows the CARS spectrum of $2 \%$ nitrogen in argon and the theoretical calculation for the same concentration. The measured spectra were normalized 


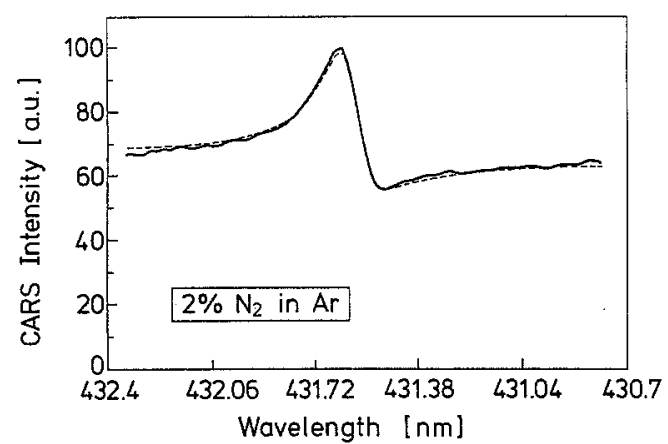

Fig. 3. Comparison of an experimental CARS spectrum from $2 \%$ nitrogen in argon with the theoretical calculation (dashed line). Both spectra were area normalized

by nonresonant CARS spectra of argon to account for the intensity distribution of the Stokes laser and the different sensitivities of the OMA-diodes. By this normalization the convolution with the Stokes intensity profile is circumvented [16]. The dispersion was measured with spectral lines of a low pressure mercury lamp at $434 \mathrm{~nm}$, giving $1.21 \mathrm{~cm}^{-1}$ per pixel at $431 \mathrm{~nm}$. The Voigt profile (FWHM $5 \mathrm{~cm}^{-1}$ ) used for convolution of the calculated spectra was at first also determined by means of these lines but we found better agreement by fitting the Voigt profile to a room temperature CARS spectrum of nitrogen measured in air. The nonlinear least-squares fitting program used for this purpose is capable of fitting up to 6 parameters like temperature, concentration, frequency, simultaneously. It should be noted that the spectra were - due to the low resolution - sensitive to the Voigt profile. In our calculations we used the Raman cross section given in [17], corrected for our Stokes laser wavelength. The Raman cross section at $540 \mathrm{~nm}$ is about 1.6 times the cross section at $607 \mathrm{~nm}$, resulting in a factor of 2.5 increase in CARS-intensity.

As shown in Fig. 3, good agreement between measurement and calculation was obtained. Best overall agreement (up to a relative nitrogen concentration of $20 \%$ in argon) was found by increasing the nonresonant susceptibilities given in [18] by $9 \%$. This can be explained by the dispersion of $\chi^{\mathrm{NR}}[19]$, because the effective frequency is higher compared to the conditions of [18]. However the increased $\chi^{\mathrm{NR}}$ is within the uncertainties of both [18] and our measurement.

\section{Single-Pulse Temperature Measurements}

To acquire CARS spectra at high repetition rates, the reading and digitizing time of the OMA must be taken into account. When all 700 (intensified) diodes are read out and digitized, one scan requires $16 \mathrm{~ms}$. The addition of two to three "cleaning scans", necessary to remove remaining electrical charge and to prevent carry-over, leads to a maximum data acquisition rate of about $15 \mathrm{~Hz}$. However by a so-called "fast-access"mode, diodes can be read out without digitizing. The fast-access requires $1 \mu \mathrm{s}$ per diode whereas normal access (read out and conversion of the analog signal to a digital value) takes place in $16 \mu$ s. Consequently the acquisition time per spectrum can be lowered by using fast-access for diodes which do not cover the spectral region of interest.

With the given spectral dispersion, 100 channels of the diode array were illuminated by the resonant $Q$-branch CARS spectrum of nitrogen (vibrational bands $v=0$ to $v=3$ ), which results in a total exposure time of $12 \mathrm{~ms}$ (including three cleaning scans). In fact the third cleaning scan removed only minor signals; after blocking the Stokes beam $1.7 \%$ of the total intensity was counted in the first, $0.78 \%$ in the second and $0.4 \%$ in the third cleaning scan. For all measurements the detector was gated with a pulse width of $10 \mathrm{~ns}$ which was sufficient to cover the temporal length of the CARS pulse.

With such an OMA timing, single-pulse CARS spectra of nitrogen were measured in the furnace. All single-pulse spectra were analyzed using a precalculated library of theoretical spectra with a horizontal frequency shift, overall normalizing factor and temperature as fit parameters. A peak normalization was applied. The computer code uses the FWHM of the measured spectra and searches in an adjustable vicinity of the theoretical spectrum with the same FWHM for the least-squares minimum. The computational and data transfer time as well as the necessary storage capacity and computer memory was considerably reduced by using 16 bit integer numbers for the normalized and calculated spectra. The time required per spectral fit was approximately three seconds.

Figure 4 shows temperatures measured by singlepulse CARS spectra versus the reading of a ther-

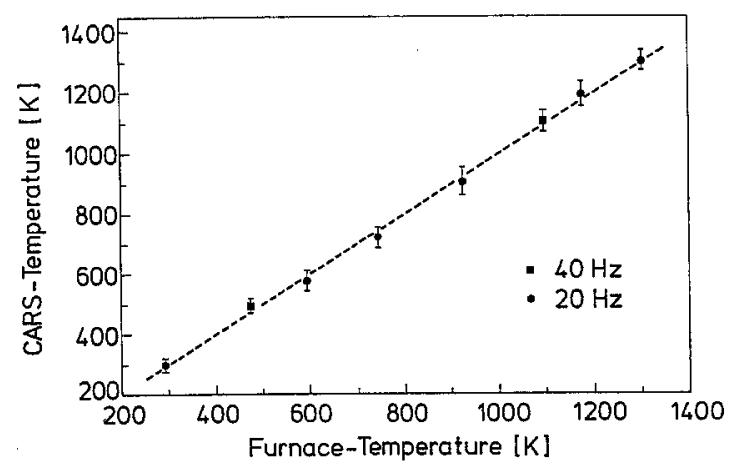

Fig. 4. Comparison of temperatures derived from single-shot CARS spectra with thermocouple-temperatures. The error bars indicate two standard deviations from the average temperature 

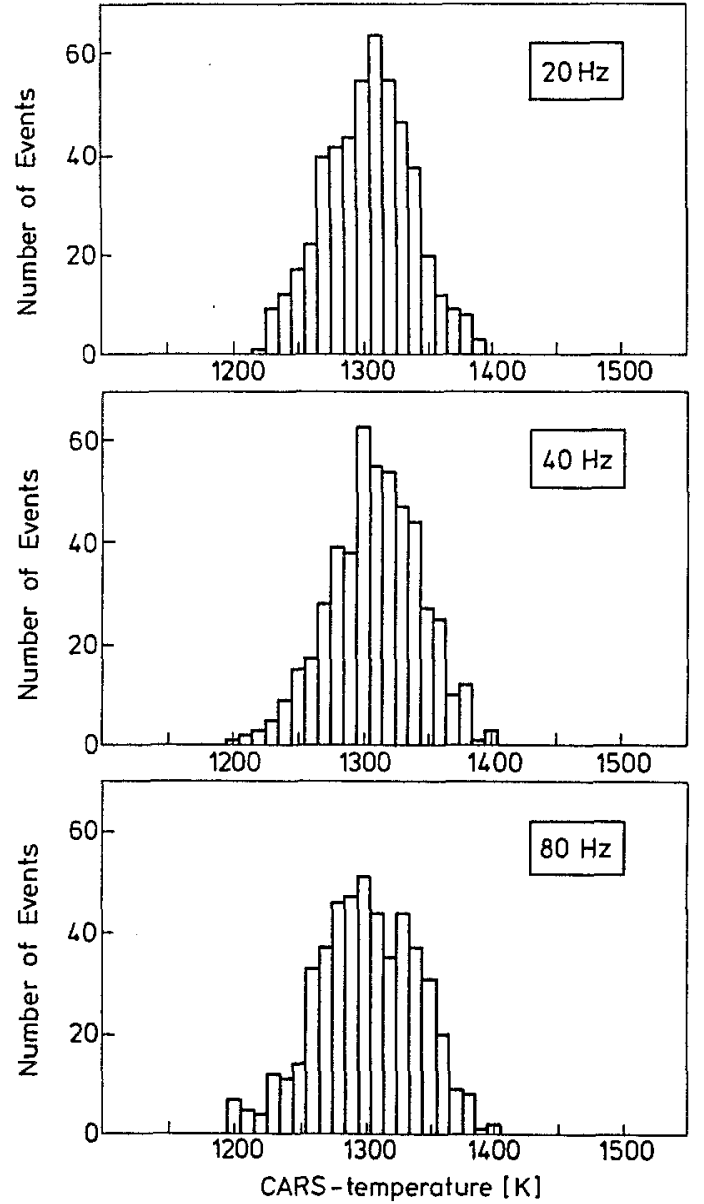

Fig. 5. Temperature histograms measured by sets of 500 singleshot CARS spectra at a furnace temperature of $1300 \mathrm{~K}$ for different repetition rates

mocouple placed close to the CARS probe volume. The error bars denote two standard deviations from the average temperature of sets of 500 spectra measured at $20 \mathrm{~Hz}$. As can be seen from Fig. 4, for all furnace temperatures the average value of the temperature histograms is within $25 \mathrm{~K}$ of the thermocouple reading. We found a small increase in the width of the histograms with furnace temperature $(24 \mathrm{~K}$ at room temperature, $35 \mathrm{~K}$ at $1300 \mathrm{~K}$ ). This might be explained by an increased amount of turbulence at high furnace temperatures. Figure 5 shows the temperature histograms for a furnace temperature of $1300 \mathrm{~K}$ for pulse repetition rates of 20,40 , and $80 \mathrm{~Hz}$. The histograms broaden slightly with repetition rate. We attribute this broadening to increased Stokes noise, caused by the fixed dye flow speed which might be still too low at high pulse repetition rates.

To study the temperature histograms in more detail, the spectral single-shot noise of both nonresonant and resonant CARS spectra was measured, the latter at a furnace temperature of $1300 \mathrm{~K}$. The percen- tage noise was obtained according to [20]. We measured $3.6 \%$ noise for a set of 500 CARS spectra of argon within a spectral interval of $120 \mathrm{~cm}^{-1}$. The noise in the resonant spectra was measured to be $5.5 \%$ (spectral interval $50 \mathrm{~cm}^{-1}$ ), increasing slightly with repetition rate. From the semiquantitative theory outlined in [21] we calculate $4.2 \%$ noise in nonresonant and $6.3 \%$ noise in resonant spectra.

With a turbulent stoichiometric Bunsen-flame located at the CARS probe volume, we measured temperature histograms for several repetition rates and fixed fuel/oxidizer flows. The spectra were recorded in the post-flame region approximately $10 \mathrm{~cm}$ above the burner surface. Figure 6 shows a normalized single-pulse CARS spectrum measured at $80 \mathrm{~Hz}$ together with the library spectrum of $2070 \mathrm{~K}$. Calculating this library of CARS-spectra we used a constant value of $1.2^{*} \chi_{\mathrm{N}_{2}}^{\mathrm{NR}}$. It has been shown that the nonresonant susceptibility in stoichiometric flames does not depend strongly on the chemical composition [2]. Single-pulse intensities of the spectra were about 700 counts at maximum. Analyzing sets of 500 spectra at a time, we obtained temperature histograms as shown in Fig. 7. As can be seen, the width of the temperature histogram measured in the turbulent flame was about $70 \mathrm{~K}$, which is about twice the width measured for isothermal conditions. The average temperature and width of the

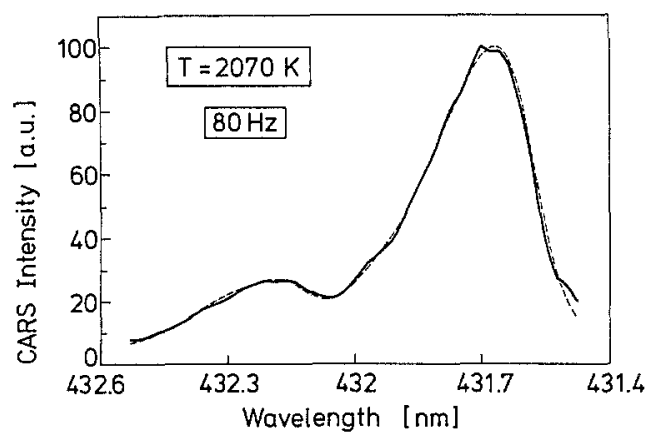

Fig. 6. Typical single-shot CARS spectrum measured at $80 \mathrm{~Hz}$ in a turbulent flame and the result of the library fit for a temperature of $2070 \mathrm{~K}$ (dashed line)

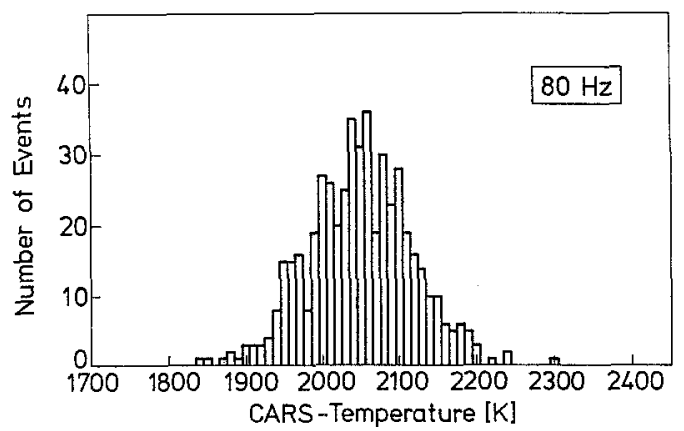

Fig. 7. Temperature histogram measured in a turbulent flame 
temperature histograms measured in the flame was independent of pulse repetition rates. About ten percent of the measured spectra were obviously distorted. Several effects could lead to such distortions: integrated CARS measurements through a region of high temperature gradients, irregular Stokes laser spectrum and beam bending. However, we did not apply a spread test [22] to remove such spectra.

\section{Summary}

We have performed single-shot BOXCARS temperature measurements for pulse repetition rates of up to $80 \mathrm{~Hz}$ using a combination of an excimer laserpumped CARS apparatus, a low dispersion detection system and for the sake of a high-speed read-out procedure, evaluating only those data carrying spectral information.

With dispersion corrected theoretical data, we could simulate the CARS spectra of nitrogen/argon mixtures satisfactorily. Even with relatively low spectral resolution, we detect sufficient sensitivity of the spectral shape to measure probability density functions of the temperature. From temperature histograms measured under stable and well-defined temperature conditions we obtained fluctuations of $\pm 50 \mathrm{~K}(90 \%$ confidence intervall) from the furnace temperature and agreement between the average temperature and the thermocouple reading within $25 \mathrm{~K}$. The obtained single-shot noise in resonant and nonresonant spectra is somewhat lower than predicted from a semiquantitative theoretical model. Regarding the low spectral noise measured, we conclude that the application of a simultaneous referencing scheme would lead only to a slight improvement.

With the CARS apparatus described here, large data sets of up to nearly 1000 spectra can be measured and analyzed at once. Such a large number of data is necessary in strongly turbulent media, where the temperature fluctuations cover a much wider range. We estimate the maximum possible rate of temperature measurements with a more advanced excimerlaser, allowing higher pulse repetition rates to be about $110 \mathrm{~Hz}$.

Up to now practical applications of excimer laserpumped CARS systems have been restricted due to relatively low pump and Stokes laser intensities. However, with recently developed high power excimer lasers which deliver up to $1 \mathrm{~J}$ pump energy per pulse, this drawback will disappear so that excimer lasers can be applied to techniques which necessiate higher pump energies such as three-colour CARS [23] or multicolour CARS techniques [24]. In addition, the possibility of external triggering of the excimer laser will allow very easy synchronization between the CARS system and the device under investigation.

Acknowledgements. The authors thank J. Hori for technical assistance and Y. Irie for his support. B. Lange wishes to express his thanks to MHI for the hospitality and aid during his stay in Japan.

\section{References}

1. A.C. Eckbreth, G.M. Dobbs, J.H. Stuflebeam, P.A. Tellex: Appl. Opt. 23, 1328 (1984)

2. R.J. Hall, L.R. Boedecker: Appl. Opt. 23, 1340 (1984)

3. M. Péalat, P. Bouchardy, M. Lefebvre, J.-P. Taran: Appl. Opt. 24, 1012 (1985)

4. R.L. Farrow, R. Trebino, R.E. Palmer: Appl. Opt. 26, 331 (1987)

5. D.A. Greenhalgh, F.M. Porter, W.A. England: Comb. Flame 49, 232 (1983)

6. S. Fuji, M. Gomi, Y. Yin: Comb. Flame 48, 232 (1982)

7. E. Diessel, Th. Dreier, B. Lange, J. Wolfrum: In preparation

8. I. Plath, W. Stricker: Proc. 3. TECFLAM-Seminar: Flammenforschung: Diagnostik und Modelle, pp. 31-46 (1987)

9. R.J. Hall, J.F. Verdieck, A.C. Eckbreth: Opt. Commun. 35, 69 (1980)

10. D.A. Greenhalgh: In Advances in Non-linear Spectroscopy, eds. R.J.H. Clarke, R.E. Hester (Wiley, New York 1988) pp. 193-248

11. M.L. Koszykowski, R.L. Farrow, R.E. Palmer: Opt. Lett. 10, 478 (1985)

12. D.A. Greenhalgh, R.J. Hall: Opt. Commun. 57, 125 (1984)

13. R.E. Teets: Opt. Lett. 9, 226 (1984)

14. R.L. Farrow, L.A. Rahn: J. Opt. Soc. Am. B 2, 903 (1985)

15. A. Gierulski, M. Noda, T. Yamamoto, G. Marowsky, A. Slenczka: Opt. Lett. 12, 608 (1987)

16. W. Kreutner, W. Stricker, Th. Just: Appl. Spectrosc. 41, 98 (1987)

17. H.W. Schrötter, H.W. Klöckner: In Raman Spectroscopy of Gases and Liquids, ed. A. Weber (Springer, New York 1979) pp. 123-201

18. R.L. Farrow, R.P. Lucht, L.A. Rahn: J. Opt. Soc. Am. B 4, 1241 (1987)

19. G.J. Rosasco, W.S. Hurst: J. Opt. Soc. Am. B 3, 1251 (1986)

20. D.A. Greenhalgh, S.T. Whittey: Appl. Opt. 24, 907 (1985).

21. S. Kröll, M. Aldēn, T. Berglind, R.J. Hall: Appl. Opt. 26, 1068 (1987)

22. D.R. Snelling, G.J. Smallwood, R.A. Sawchuk, T. Parameswaran: Appl. Opt. 26, 99 (1987)

23. R.P. Lucht: Opt. Lett. 12, 78 (1987)

24. A.C. Eckbreth, T.J. Anderson, G.M. Dobbs: Appl. Phys. B 45, 215 (1988) 